\title{
WOX and KNOX transcription factors in symbiotic nodule development
}

\author{
Lebedeva M.A.*, Azarakhsh M., Dodueva I.E., Lutova L.A. \\ Department of Genetics and Biotechnology, St. Petersburg State University, St. Petersburg, Russia \\ *e-mail:mary_osipova@mail.ru
}

WOX and KNOX transcription factors (TFs) regulate different aspects of plant development, including meristem formation and maintenance. Their involvement in diverse developmental programs allows unraveling the evolution of regulatory mechanism that accompanies the formation of new organs in plant. We studied the role of WOX and KNOX TFs in symbiotic nodule formation in Medicago truncatula. WOX TFs are known as key regulators of plant meristems working together with CLV1-like receptor and CLE peptides. We have analyzed their role in nodulation and found that WOX5 gene is involved in nodule development. Moreover, using sunn supernodulating mutant we found that WOX5 expression is dependent on CLV1-like kinase SUNN that regulates nodule number in $M$. truncatula. The role of other members of WOX family in nodulation has been studied as well.

Together with WOX family, KNOX TFs regulate diverse developmental programs in plants. Specifically, in the shoot apical meristem KNOX TFs activate the expression of isopentenyl transferase (IPT) genes involved in cytokinin biosynthesis. We found that nodule development is regulated by a member of KNOX TF family KNOX3. We found that in developing nodules KNOX3 activates the expression of genes involved in cytokinin biosynthesis, in particular, genes of IPT and $L O G$ families, by direct binding to their regulatory sequences.

To summarize, studying the role of WOX and KNOX TFs in nodule development suggests that different developmental programs in plants are regulated by common regulatory modules involved WOX-CLV system and KNOX TFs with their target genes. Acknowledgements: This work was supported by Russian Scientific Foundation project No. 16-16-10011 and RFBR grant No. 17-04-01708. 